\title{
NOUVELLES DIVERSES
}

\section{L'accident de Three Mile Island \\ et \\ le taux de mortalité infantile en Pennsylvanie}

En janvier 1980, E. J. Sternglass, professeur de Physique radiologique à l'École de Médecine de Pittsburg, bien connu pour ses options antinucléaires, présentait à Tel Aviv une de ces communications à sensation dont il a l'habitude. Aux États Unis, cette étude n'a joui d'aucun crédit car les libertés que l'auteur a toujours prises avec les règles d'interprétation des données statistiques et la précipitation avec laquelle il utilise des informations mal établies sont légendaires.

Dans cette communication, E. J. STERnglass essayait de prouver que, dans l'État de Pennsylvanie, la mortalité des enfants de moins de 1 an (mortalité infantile) avait significativement augmenté dans les 5 mois qui suivirent l'accident de réacteur de Three Mile Island (TMI) survenu le 28 mars 1979. Cette augmentation était attribuée à la dose qu'aurait pu recevoir la glande thyroïde des enfants se trouvant dans les 5 derniers mois du stade fœtal au moment où furent rejetés dans l'atmosphère des gaz rares et une certaine activité d'iode radioactif. Ces rejets se situent au cours des tout premiers jours qui suivirent l'accident.

Les informations statistiques sur lesquelles E. J. STERnGLass s'appuyait pour justifier sa thèse étaient extraites des rapports statistiques mensuels publiés par le Centre national de statistiques sanitaires (niveau fédéral) et comprenaient, pour 1979, le nombre de cas et le taux de mortalité infantile dans l'État de Pennsylvanie pour les 7 premiers mois de l'année.

Il est important de noter que :

1. Ces informations étaient entachées d'une erreur commise au niveau fédéral dans la retranscription des chiffres transmis par les autorités sanitaires de l'État de Pennsylvanie : le nombre de cas de mortalité infantile pour le mois de juillet 1979 était de 185 et non de 271 .

2. Elles étaient établies, comme cela est normal, sur la population résidant dans l'État de Pennsylvanie au cours du mois pour lequel les services sanitaires établissaient les données statistiques. Or, cette population qui est affectée par les 
changements de résidence, relativement fréquents aux U.S.A., n'est pas la même d'un mois à l'autre et surtout n'est pas représentative de la population résidant en Pennsylvanie les 28, 29 et 30 mars 1979. On sait, en effet, qu'un certain nombre de familles ont été s'établir dans un autre état à la suite de l'accident de TMI.

Une étude approfondie, présentant toutes les garanties de rigueur scientifique et s'attachant à suivre la population présente dans l'État de Pennsylvanie au cours des derniers jours de mars, s'imposait donc pour apprécier la thèse avancée par E. J. STERnGLASS. Cette étude fut menée par le Département de la Santé de l'État de Pennsylvanie et ses résultats ont été publiés le 20 mars 1981.

On y trouve toutes les dernières données concernant les taux de mortalité infantile (nombre de décès d'enfants de moins de 1 an sur 1000 enfants nés vivants), de mortalité dans les 28 premiers jours de vie (neonatal death), de mortalité fœtale (avec ou sans les avortements), mois par mois pour 1979, trimestre par trimestre pour 1977, 1978 et 1979 et ce, pour les populations résidant dans quatre régions géographiques :

- l'ensemble de l'État de Pennsylvanie;

- une région délimitée par un cercle de $18 \mathrm{~km}$ de rayon autour de TMI;

- la partie de la ville de Harrisburg, comprise dans le cercle précédemment défıni, partie où réside principalement une population noire à caractéristiques particulières;

- la région définie par le même cercle moins la partie de Harrisburg précédente.

On se limitera, ici, à présenter les résultats concernant la mortalité infantile pour l'État de Pennsylvanie et pour la région située à l'intérieur du cercle de $18 \mathrm{~km}$ de rayon centré sur TMI et on en tirera les conclusions. L'analyse des autres données conduit aux mêmes résultats.

\section{MORTALITÉ INFANTILE DANS L'ÉTAT DE PENNSYLVANIE}

La figure 1 présente l'évolution, mois par mois au cours de l'année 1979, du taux de mortalité infantile dans la population résidant dans l'État de Pennsylvanie fin mars 1979 comparée à l'évolution de ce même taux pour la population de cet État au cours de l'année 1978.

Le taux de mortalité infantile pour la population résidant en fin mars dans l'État de Pennsylvanie est resté, pratiquement durant toute l'année 1979, inférieur à ce qu'il était pour la population de cet état en 1978 .

L'allure générale des deux tracés est la même, en particulier durant les mois d'été au cours desquels E. J. STERnGLASs situait une augmentation anormale en 1979.

Le tableau ci-dessous et la figure 2 donnent les valeurs trimestrielles du taux de mortalité infantile pour la population étudiée en 1979 et pour l'État de Pennsylvanie en 1978 et en 1977. 


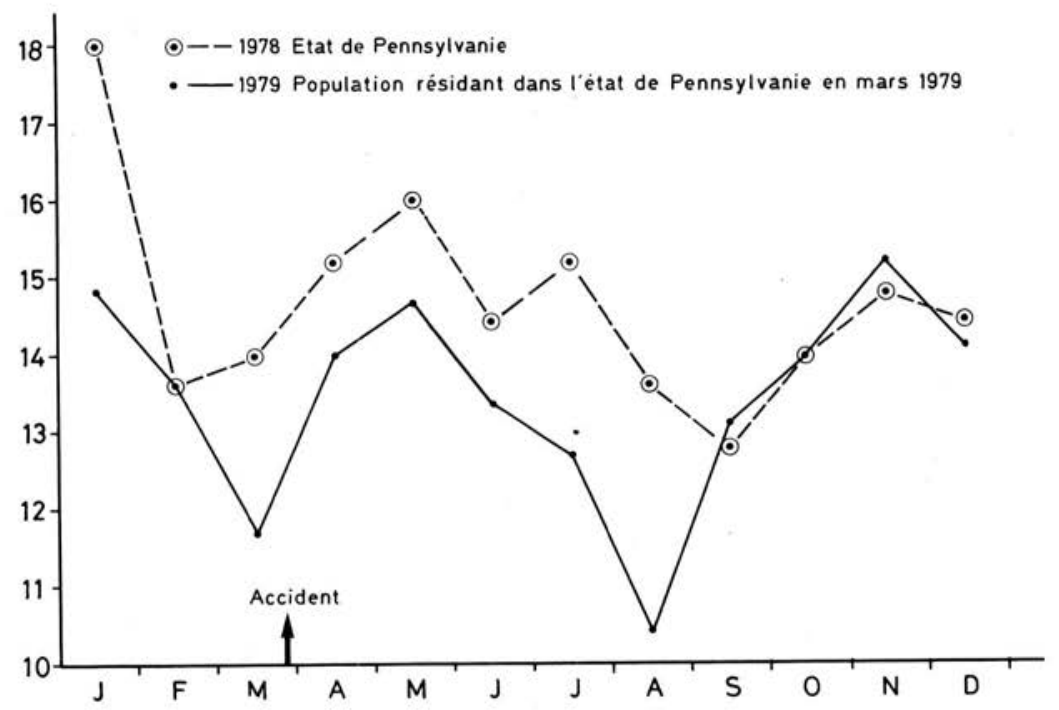

FIG. 1. - Taux de mortalité infantile mensuel (nombre de décès d'enfants de moins de 1 an sur 1000 enfants nés vivants). $\odot--1978$ État de Pennsylvanie. - — 1979 Population résidant dans l'État de Pennsylvanie en mars 1979.

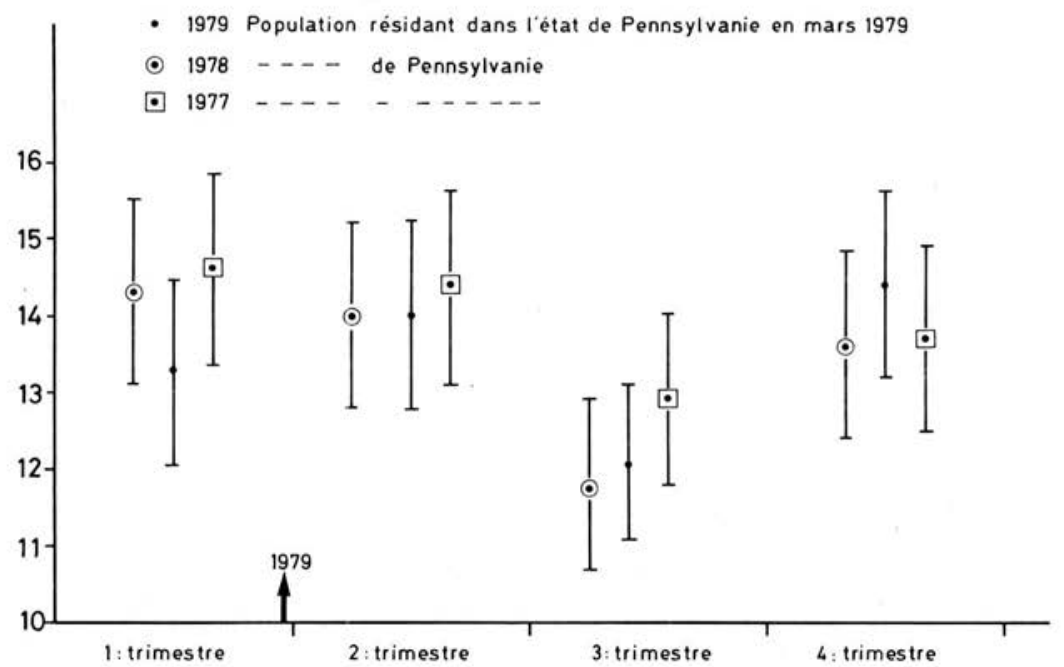

FIG. 2. - Taux de mortalité infantile trimestriel (nombre de décès d'enfants de moins de 1 an sur 1000 enfants nés vivants) • 1979 Population résidant dans l'État de Pennsylvanie en mars 1979. • 1978 Population de Pennsylvanie. 1977 Population de Pennsylvanie.

vOL. $16-\mathrm{N}^{\circ} 2$ 
Il a été possible ici de calculer les marges d'incertitude $95 \%$ à partir du nombre de cas de mortalité infantile que donne, par trimestre, le document du Département de la santé de l'État de Pennsylvanie :

\begin{tabular}{|l|c|c|c|}
\hline & 1977 & 1978 & 1979 \\
\hline $1^{\text {er }}$ trimestre. $\ldots \ldots$ & $14,7 \pm 1,2$ & $14,3 \pm 1,2$ & $13,3 \pm 1,1$ \\
$2^{\mathrm{e}}$ trimestre...... & $14,4 \pm 1,2$ & $14 \pm 1,2$ & $14 \pm 1,2$ \\
$3^{\mathrm{e}}$ trimestre...... & $12,9 \pm 1,1$ & $11,8 \pm 1,1$ & $12,1 \pm 1$ \\
$4^{\mathrm{e}}$ trimestre....... & $13,7 \pm 1,2$ & $13,6 \pm 1,2$ & $14,4 \pm 1,2$ \\
\hline
\end{tabular}

Il est clair que, pour chaque trimestre, aucune différence significative ne peut être mise en évidence entre les 3 années.

\section{MORTALITÉ INFANTILE DANS LA POPULATION RÉSIDANT A MOINS DE $18 \mathrm{~km}$ DE TMI}

Cette région comprend certains quartiers de la ville de Harrisburg habités par une population à taux de mortalité infantile souvent beaucoup plus élevé que le taux moyen pour l'État de Pennsylvanie. Le tableau ci-dessous donne pour cette région les taux de mortalité infantile trimestriels pour 1979 (résidants en mars), 1978 et 1977, et la figure 3 reprend graphiquement ces données :

\begin{tabular}{|l|c|c|c|}
\hline & 1977 & 1978 & 1979 \\
\hline $1^{\text {er }}$ trimestre. . . . . & $14,7(7,7-25)$ & $14(7,4-24)$ & $19,3(11,5-30,5)$ \\
$2^{\mathrm{e}}$ trimestre...... & $11,7(5,8-20)$ & $9,8(4,4-18,4)$ & $19,3(11,6-30,1)$ \\
$3^{\mathrm{e}}$ trimestre.. $\ldots \ldots$ & $9,2(4,2-17,4)$ & $4,9(1,5-11,2)$ & $12,7(6,7-21,7)$ \\
$4^{\mathrm{e}}$ trimestre...... & $14,7(8-24,7)$ & $15,1(8,2-25,3)$ & $13,4(7,1-23)$ \\
\hline
\end{tabular}

Ces taux ont été calculés sur un nombre de cas faible, aussi les marges d'incertitude, fondées sur la loi de Poisson, sont-elles considérables.

On peut voir que la valeur (élevée) du taux de mórtalité infantile au cours du $2^{\text {e }}$ trimestre 1979 est la même qu'au cours du $1^{\text {er }}$ trimestre avant l'accident de TMI, et que cette valeur décroît ensuite nettement au cours des 2 derniers trimestres.

D'autre part, le calcul montre qu'il n'y a aucune différence significative entre ces valeurs et celles que l'on trouve pour les trimestres correspondants en 1977 et en 1978. 


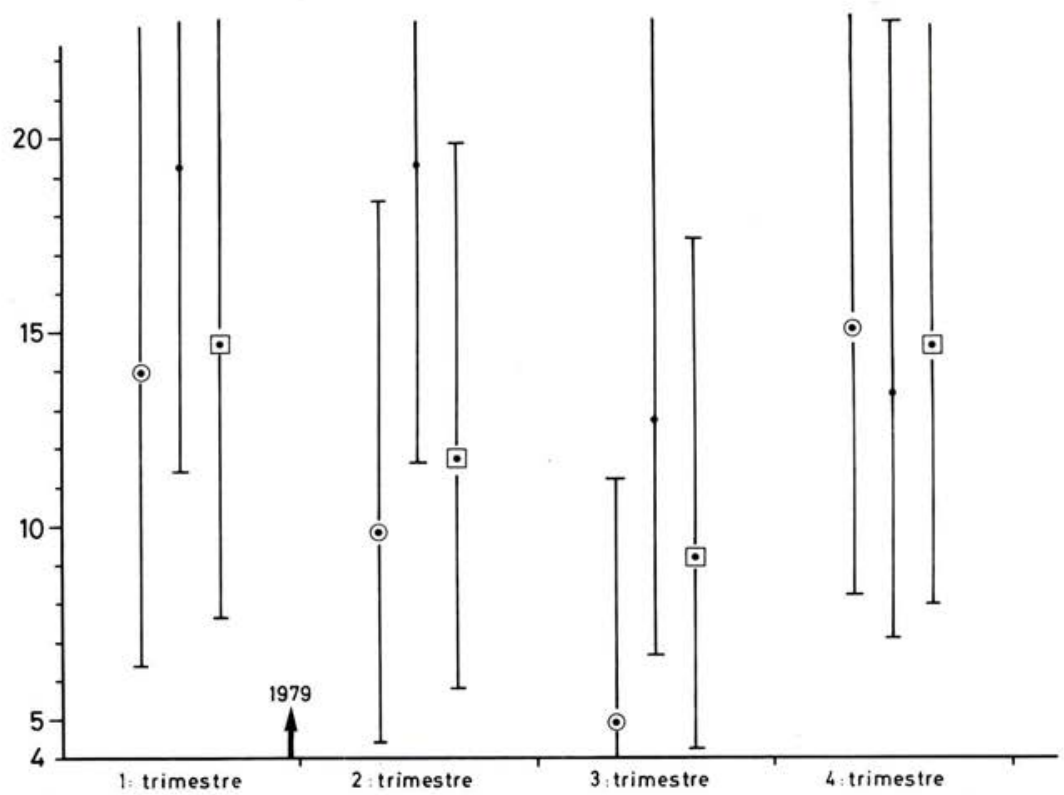

FIG. 3. - Taux de mortalité infantile trimestriel dans les $18 \mathrm{~km}$ autour de TMI. • 1979 Population résidant dans le cercle de $18 \mathrm{~km}$ de rayon en mars 1979. $\odot 1978$ Population résidant dans le cercle de $18 \mathrm{~km}$ de rayon en 1978. 1977 Population résidant dans le cercle de $18 \mathrm{~km}$ de rayon en 1977.

\section{CONCLUSIONS}

La conclusion de cette brève étude est qu'il est évident que l'accident de TMI n'a eu aucune incidence détectable sur le taux de mortalité infantile ni dans l'ensemble de l'État de Pennsylvanie, ni dans les régions les plus proches de la centrale.

E. J. Sternglass, comme beaucoup de ceux qui sont animés d'un prosélytisme militant, a utilisé en fin 1979 des données qui n'étaient pas encore parfaitement établies. Dans la hâte d'argumenter pour défendre leurs opinions, il arrive à des esprits, même formés aux disciplines scientifiques, de ne pas vérifier avec assez de soin les informations de base sur lesquelles se fonde leur démonstration.

M. DOUSSET 


\section{COMPTE RENDU RAPIDE DU BEIR III ET DE LA CONTROVERSE QU'IL A SUSCITÉE}

Le Comité scientifique de l'Académie des Sciences américaine avait publié en 1972 un rapport sur les effets biologiques des rayonnements ionisants, connu sous le nom de BEIR I $\left({ }^{1}\right)$. Depuis lors, les 22 experts constituant ce Comité ont pensé devoir réactualiser l'étude, à partir de toutes les nouvelles données disponibles. Les premières conclusions en ont été présentées à Washington en mai 1979 par son président Radford, de l’Université de Pittsburg. Un désaccord important étant apparu parmi les membres du Comité à propos des estimations d'effets somatiques au niveau des faibles doses, le président fut exclu et la parution du rapport final fut différée d'un an. C'est un panel de sept membres qui constitua le sous-comité chargé de la rédaction des effets somatiques, sous la direction du Dr J. Fabrikant, qui s'efforça de rendre compte au mieux des différents points de vue qui s'étaient préalablement exprimés.

Par contre, aucune discussion de même ampleur n'a eu lieu à propos des effets génétiques pour lesquels la relation linéaire-quadratique a été retenue par tous.

C'est ainsi que le rapport final, paru le 29 juillet $1980\left({ }^{2}\right)$, donne un éventail de valeurs de risque, pour les faibles doses, qui a été accepté à l'unanimité des membres, à l'exception de deux d'entre eux.

\section{CONClusions ESSENTIELles SUR LES EFFETS SOMATIQUeS}

Aucun désaccord n'est apparu ni à propos des résultats scientifiques ni des données épidémiologiques ou expérimentales concernant les effets des doses élevées (d'au moins une centaine de rems) délivrées à forts débits de doses. De même, l'unanimité a reconnu qu'aucune mise en évidence n'existe de l'effet nocif de l'irradiation naturelle, de l'ordre de $100 \mathrm{mrem}$ par an. Si un tel effet existait, il serait sans doute masqué par d'autres facteurs de l'environnement.

Par contre, la controverse a porté sur l'interprétation de toutes ces données et sur l'estimation du risque de l'exposition de la population à des niveaux de dose de l'ordre de $10 \mathrm{mrem}$, voire de 100 à $500 \mathrm{mrem}$ par an de rayonnements de bas TEL, se surajoutant au 100 mrem inévitables d'origine naturelle. Quant au risque des travailleurs, exposés à une dose de l'ordre de $500 \mathrm{mrem}$, voire plus, jusqu'à 5 rems par an, il est, de l'avis de la majorité, à considérer de façon distincte.

(1) BEIR : Biological effects of ionizing radiations.

$\left({ }^{2}\right)$ Voir Radioprotection, 1980, vol. $15, \mathrm{n}^{\circ} 4$, p. 248. 
Jusqu'à présent, et en accord avec toutes les autres commissions d'experts internationaux (C.I.P.R., UNSCEAR), les experts du BEIR avaient adopté la relation linéaire pour calculer les risques aux faibles doses ( $c f$. BEIR I de 1972). Le draft de 1979 reprenait les mêmes hypothèses. C'est depuis la constitution du groupe présidé par Fabrikant en mai 1979 que l'on a discuté cette hypothèse en affirmant qu'elle amenait une surestimation importante du risque aux faibles doses, du moins pour les rayonnements à faible TEL. Cette opinion fut étayée sur un grand nombre de données radiobiologiques et de résultats épidémiologiques.

Ainsi, pour la majorité des membres du Comité, il apparaît que la plupart des affections malignes radioinduites obéissent à une relation, au niveau des faibles doses et jusqu'aux doses intermédiaires, du type linéaire-quadratique, relation qui conduit à des valeurs de risque intermédiaires entre celles calculées selon la relation linéaire et celles calculées selon la relation quadratique (tableau I et figure 2).

\section{TABLEAU I}

NOMBRE DE MORTS PAR CANCER PAR MILLION DE PERSONNES EXPOSÉES À 1 REM. CALCULÉ SELON DIFFÉRENTS MODÈLES.

\begin{tabular}{|l|c|c|c|}
\hline & $\begin{array}{c}\text { Relation } \\
\text { linéaire }\end{array}$ & $\begin{array}{c}\text { Relation } \\
\text { linéaire-quadratique }\end{array}$ & $\begin{array}{c}\text { Relation } \\
\text { quadratique }\end{array}$ \\
\hline UNSCEAR 1977. & 100 & - & - \\
C.I.P.R. 1977.... & 125 & - & - \\
BEIR I (1972). . . & $115-621\left(^{1}\right)$ & $67-226\left(^{2}\right)$ & $10-28$ (Rossi) \\
BEIR III (1980)... & $150-501\left(^{1}\right)$ & (majorité) & \\
Selon Radford. ... & $405-1210$ & - & - \\
\hline
\end{tabular}

(1) Les fourchettes de valeurs different entre le BEIR I et le BEIR III par suite de données différentes concernant l'incidence spontanée de cancers entre les statistiques de 1967 (BEIR I) et celles de 1969-1971 (BEIR III).

$\left({ }^{2}\right)$ Ces valeurs englobent tous les modes d'irradiation (unique ou chronique) ainsi que tous les modes de calcul (risque absolu ou relatif, celui-ci tenant compte de l'âge à l'exposition).

Ainsi aboutit-on à des valeurs de risque approximativement deux fois plus faibles que celles données en 1972, par le BEIR I. Deux autres membres du Comité sont toutefois demeurés en désaccord avec ces conclusions. Ils ont exprimé leur opinion dans des rapports annexés au rapport principal et que nous allons examiner tout à tour.

\section{RAPPORTS DISSIDENTS}

Rapport de Radford: L'auteur, ancien président du Comité BEIR, explique les points essentiels de discorde apparus au sein du sous-comité sur les effets somatiques du BEIR III :

$1^{\circ}$ sur le choix approprié de la relation dose-effet, permettant l'extrapolation aux faibles doses des données obtenues à fortes doses; 
$2^{\circ}$ sur la sélection convenable des données humaines disponibles.

Pour lui, l'utilisation de la relation linéaire et sans seuil constitue une approximation excellente, en accord avec les données admises de radiobiologie fondamentale ( fig. 1).

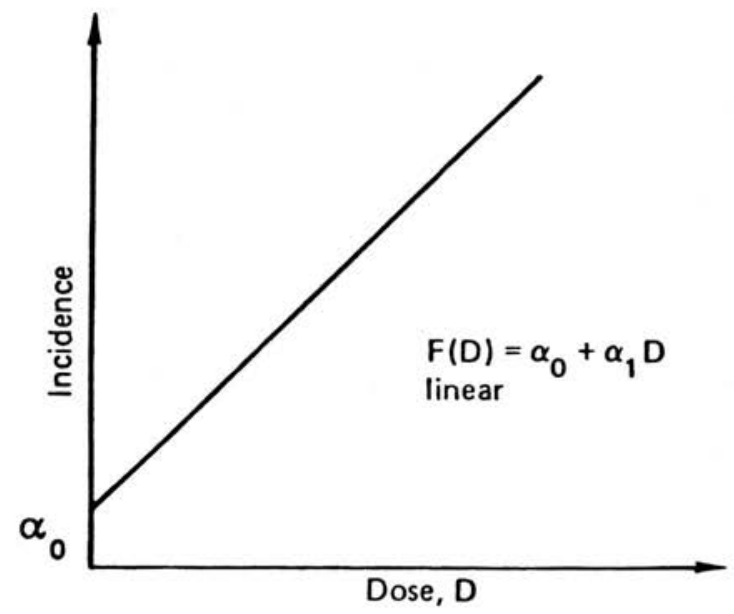

FIG. 1

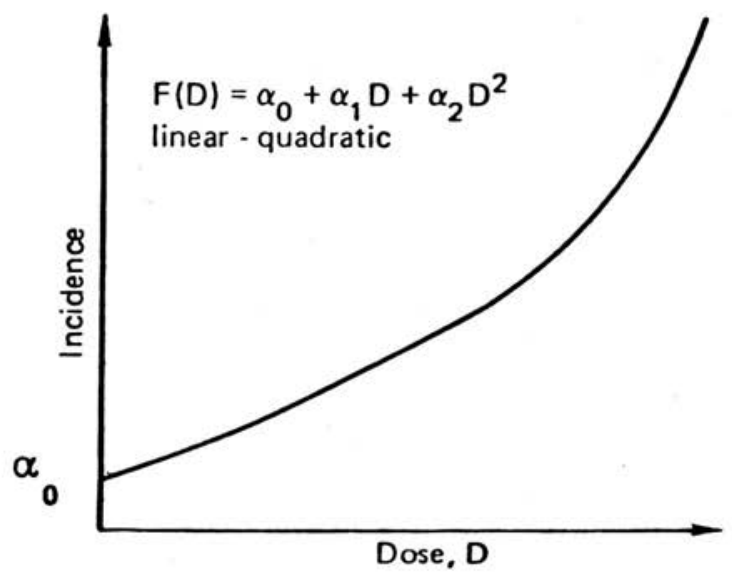

FIG. 2

Emprunté au BEIR III, (fig. II. 2) p. 28

La version définitive du BEIR III lui semble sous-estimer de façon significative le risque de cancer radioinduit, en négligeant l'effet des forts débits de doses de rayonnements à faible TLE sur la survie cellulaire, laquelle est liée à la probabilité d'induction cancéreuse au cours du temps. La valeur du risque la 
plus correcte est, pour lui, sur la durée de vie, de 260 à 800 cas de cancers par million de personnes et par rad chez les hommes, et de 550 à 1620 cas chez les femmes, valeur cinq fois supérieure à celle indiquée dans le rapport. Il critique, enfin, la position de Rossi, partisan de la relation quadratique, qui lui paraît contraire à tous les résultats d'études théoriques, expérimentales et épidémiologiques.

Rapport de Rossi : Rossi, lui, s'appuyant sur les résultats des observations des survivants japonais, insiste beaucoup sur le divorce existant entre l'effet des deux types de rayonnements, selon leur TEL, gamma d'une part et neutrons

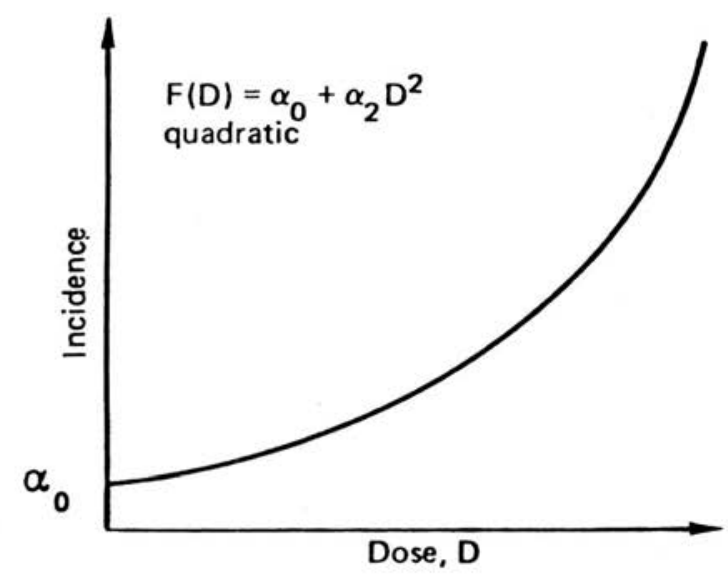

FiG. 3

Emprunté au BEIR III, (fig. II.2) p. 28

d'autre part. Dans le cas des photons, conformément à l'ensemble des résultats de la radiobiologie et de l'épidémiologie, il pense que les estimations de risque, selon les relations linéaire ou linéaire-quadratique, ne sont pas les plus plausibles. Pour lui, pour ce type de rayonnements, c'est la relation quadratique qui serait la plus adaptée ( fig. 3). Cette relation conduit à des valeurs de risque très petites aux faibles doses et qui se rapprochent de zéro aux doses $\leqq 1 \mathrm{mrem}$. Par contre, il demeure partisan de la relation linéaire pour les rayonnements à TEL élevé, tels les neutrons. Cela entraîne de grands écarts d'effets aux faibles doses, c'est-à-dire un EBR des neutrons très élevé, et d'autant plus élevé que la dose s'amenuise. La figure jointe visualise ces écarts. Rossi insiste beaucoup, ensuite, sur les conséquences de ce mode d'évaluation du risque, pour les rayonnements à TEL faible, et notamment sur l'absence de validité d'aucun des concepts de radioprotection qui découlent logiquement de la loi linéaire et notamment du risque ramené au rem, de l'homme-rem, du dommage collectif, etc. 


\section{CONCLUSION}

L'intérêt du rapport BEIR est donc de donner tous les avis ainsi que toute l'argumentation attachée à chacune des positions. De toute façon, à la majorité, le choix de la relation linéaire est rejeté comme modèle le plus adapté à l'estimation des risques au niveau des faibles doses $\leqq 25$ rems et des faibles débits de dose, pour les rayonnements à faible TEL. Ainsi se trouve remis en question tous les modes classiques de calcul de dommage sur la population des travailleurs ou sur la population toute entière.

\section{S. VIGNES}

\section{INCENDIE D'UN SILO DE STOCKAGE A LA HAGUE}

Le 6 janvier dernier, un local de stockage de chemises de graphite a été le siège d'un incendie. La presse a très largement couvert cet événement dont il convient d'évaluer les conséquences au strict plan de la radioprotection, en dehors de tout aspect économique, politique et social. Dans l'attente d'un document plus complet, on peut résumer comme suit les conclusions d'une commission d'enquête mise en place par le CHS (Comité d'hygiène et de sécurité) du Centre de La Hague :

- Conséquences radiologiques pour le personnel : 5 agents ont reçu une irradiation externe, dont un à $5,7 \mathrm{rem}$ et les autres entre 200 et $400 \mathrm{mrem}$. 35 agents ont fait l'objet d'une décontamination au service médical (contamination légère). Les contrôles par antropogammamétrie ont décelé la présence de ${ }^{137} \mathrm{CS}$ représentant au maximum $1 / 1000$ de la DMA.

- Conséquences pour l'environnement : des campagnes de mesures ont été lancées sur le sol, les végétaux, les bâtiments situés sous le vent, et dans l'atmosphère; aucune contamination du sous-sol n'a été mise en évidence.

La commission d'enquête a émis des recommandations portant sur l'amélioration du stockage, le renforcement des consignes d'exploitation, de sécurité-incendie, de contrôle radiologique, et d'information sur site et hors site.

\section{CAMPAGNE D'ÉTALONNAGE DE DÉTECTEURS}

Le L.C.I.E., Laboratoire central des industries électriques, annonce le lancement d'une campagne d'étalonnages groupés pour les détecteurs $\mathrm{X}$ ou $\mathrm{Y}$ utilisés en radioprotection.

Les établissements et entreprises ayant à satisfaire aux prescriptions du décret $\mathrm{n}^{\circ}$ 67-228 du 15 mars 1967 doivent, notamment, étalonner périodiquemment leurs appareils.

Le L.C.I.E. organise de mai à novembre 1981 plusieurs séries de mesures, et fournit les conditions matérielles et financières d'inscription, selon le type d'appareil.

Pour tous renseignements, s'adresser à M. Pierré, L.C.I.E., B.P. $n^{\circ}{ }^{8,92260}$ Fontenay-aux-Roses. 


\section{COMPTE RENDU DU COLLOQUE INTERNATIONAL SUR LA DOSIMÉTRIE BIOMÉDICALE, PARIS, 27-31 OCTOBRE 1980}

Du 27 au 31 octobre 1980 s'est tenu à Paris un Colloque international de dosimétrie biomédicale, organisé par l'Agence Internationale de l'Énergie Atomique en collaboration avec l'Organisation Mondiale de la Santé et sur invitation du Gouvernement Français; l'organisation matérielle du colloque avait été confiée au Laboratoire de Métrologie des Rayonnements Ionisants (C.E.A./O.R.I.S.). Plus de 200 spécialistes venus de 43 pays et de 3 organisations internationales ont participé aux différentes sessions :

aspects généraux de la dosimétrie, aspects physiques et biomédicaux particuliers, détermination de la dose absorbée, normalisation et étalonnages des systèmes dosimétriques, développement des systèmes dosimétriques.

La dosimétrie des rayonnements, qui a pour objet essentiel la détermination de la dose absorbée par la matière irradiée, intervient dans toute application des rayonnements ionisants, car l'effet produit dans la matière est en étroite relation avec l'énergie déposée par les particules ionisantes. La nécessité d'une bonne dosimétrie est,évidemment,d'une importance particulière dans le domaine de la santé de l'homme, qu'il s'agisse de la prévention et du contrôle des risques liés à l'industrie nucléaire, de l'utilisation par le médecin, à des fins de diagnostic, de substances radioactives (médecine nucléaire) ou de faisceau de rayon $\mathrm{X}$, ou encore de l'application de faisceaux de photons, d'électrons, de neutrons en radiothérapie.

L'importance croissante que le public attache à l'utilisation sûre des rayonnements et l'affınement des connaissances et des moyens mis en œuvre en radiothérapie ont, ces dix dernières années, amené les chercheurs et les ingénieurs à redoubler d'efforts pour mettre au point des appareils plus fiables, efficaces et sûrs et améliorer encore la précision des mesures de doses pour tous les rayonnements utilisés en biologie et en médecine.

La quarantaine de communications présentées et les discussions qu'elles ont entrainées ont fait ressortir un certain nombre de thèmes dominants parmi lesquels les trois suivants sont à noter :

- La récente généralisation du système international d'unités ayant provoqué un ré-examen fondamental des grandeurs dosimétriques à considérer lors de l'étalonnage des instruments de-mesure, diverses propositions ont été avancées par des représentants de laboratoires nationaux de métrologie pour le remplacement de la grandeur "exposition" (unité $\mathrm{SI}=$ le coulomb par kilogramme) par une grandeur, « kerma » ou " dose absorbée » (unité : le joule par kilogramme, dont le nom spécial est le " gray »), plus proche de la grandeur utile en dosimétrie appliquée. Ce problème est d'une grande importance en dosimétrie médicale car l'affınement des techniques radiothérapeutiques a entraîné un besoin accru de précision dans la connaissance des doses absorbées par les divers tissus irradiés lors d'un traitement.

- L'utilisation de faisceaux de neutrons rapides (issus de cyclotrons) en radiobiologie et en radiothérapie n'est pas sans poser de problèmes de mesure 
tant en ce qui concerne les aspects théoriques que l'instrumentation. Les utilisateurs sont dans ce domaine plus démunis que dans celui plus classique des photons et des électrons car les étalons de références sont encore quasiinexistants. Un effort remarquable de normalisation est cependant poursuivi aux États-Unis d'Amérique et en Europe, au sein de groupes de travail mis en œuvre par les physiciens médicaux concernés. Les études engagées dans certains laboratoires nationaux (dont le L.M.R.I., laboratoire primaire du Bureau National de Métrologie) devraient permettre dans quelques années la mise en place d'un système de références dosimétriques cohérent adapté à ce domaine particulièrement complexe.

- Dans le domaine de la coopération internationale, les efforts sont poursuivis en direction des pays en voie de développement. L'A.I.E.A. et l'O.M.S. ont mis en place un réseau mondial de laboratoires secondaires de dosimétrie, dont l'importance a été soulignée et pour lequel il a été fait appel à une participation encore plus active des laboratoires nationaux des pays industrialisés.

\author{
J. P. Simoen, M. CANCE, \\ J. Caumes, K. Steinschaden
}

\title{
COMPTE RENDU SUR LE « DEUXIÈME SYMPOSIUM SPÉCIAL SUR L'IRRADIATION NATURELLE ", BOMBAY, 19-23 JANVIER 1981
}

Les congrès internationaux ayant trait à l'irradiation naturelle sont peu nombreux. Celui de Bombay s'inscrivait dans la série de ceux organisés, directement ou indirectement, par J. ADAMs, Professeur de géologie à Houston. Il faisait suite au premier symposium spécial qui s’était tenu à Poços de Caldas, Brésil, en juin 1975. Cependant, alors que le congrès de Poços de Caldas était uniquement consacré aux zones à activité naturelle élevée, celui de Bombay avait un caractère plus large puisque pratiquement tous les aspects de l'irradiation naturelle ont été abordés.

Entièrement organisé par les Indiens, il a accueilli environ cent cinquante participants en provenance d'une vingtaine de pays et a fait l'objet d'une centaine de communications réparties selon les rubriques suivantes : zones à activité naturelle élevée; environnement radioactif naturel; techniques de mesures; accroissement de l'irradiation naturelle du fait de la technologie; irradiation naturelle à l'intérieur des habitations; le radon et ses descendants dans l'air ambiant; application des mesures d'activité naturelle en géophysique.

Les points forts de ce symposium me semblent avoir été les suivants :

$1^{\circ}$ L'importance accordée aux mesures de radon dans de nombreux pays. Cette importance est tout à fait justifiée puisque l'inhalation de radon et de ses descendants à vie courte à l'intérieur des habitations est responsable d'environ la moitié de la dose totale (somme de l'irradiation externe et des doses dues à l'inhalation et à l'ingestion de tous les radionucléides naturels) reçue par les 
populations. Du fait que la politique de conservation d'énergie conduit à la limitation du taux de ventilation dans les bâtiments et, par conséquent, toutes choses égales par ailleurs, à l'augmentation des concentrations en radon, l'intérêt grandissant manifesté pour les mesures de radon est peut être encore loin d'avoir atteint son maximum. La principale difficulté de l'interprétation des mesures de radon dans les habitations réside dans leur variabilité : pour une habitation donnée, les concentrations offrent en général des variations journalières marquées et, d'une habitation à l'autre, les variations sont également très nettes et pas nécessairement corrélées aux niveaux d'irradiation externe. Aussi les études tendent-elles maintenant à s'orienter vers la mesure des sources (taux d'émanation des murs et du sol, concentration dans l'eau) et vers l'analyse des mécanismes permettant d'interpréter les niveaux observés.

$2^{\circ}$ L'orientation vers la réglementation de certaines expositions à des sources d'irradiation naturelle. Ce problème a été évoqué par des orateurs américains, polonais et suédois et a surtout été développé par H. JAMMET, Directeur au C.E.A., qui a exposé les différentes approches possibles du problème et a jeté les grandes lignes d'un système réglementaire compatible avec les recommandations de la C.I.P.R.

$3^{\circ}$ Le renouveau des études sur les zones à activité naturelle élevée. Ce renouveau est très net en Inde où les recherches dans les zones à activité élevée du Kerala et de Tamil Nadu portent, en particulier sur l'analyse complète des voies d'exposition. Par ailleurs, plusieurs équipes allemandes travaillent sur des zones de leur pays présentant des niveaux d'activité naturelle modérément élevés.

Si le symposium a présenté des points forts, il a aussi permis de constater que certains domaines d'études ne recevaient pas, à l'heure actuelle, l'intérêt qu'ils semblent mériter. Il s'agit, entre autres :

(a) des mesures faites sur l'homme : seul WRENN (Université d'Utah) a présenté des résultats très intéressants sur les concentrations en uranium et en thorium dans divers tissus et organes humains;

(b) des études sur les minerais phosphatés et leurs dérivés : les minerais phosphatés d'origine marine sont, en général, riches en uranium; leur extraction conduit principalement à la fabrication d'engrais et à la production de sulfate de calcium qui peut éventuellement être utilisé comme plâtre. Les études consacrées à ces questions paraissent actuellement marquer le pas;

(c) les mesures de thoron dans les habitations: les quelques mesures disponibles semblent montrer que ses niveaux (ainsi que les doses résultantes) sont bien plus faibles que ceux du radon mais les résultats sont assez contradictoires et mériteraient d'être confirmés par d'autres séries de mesures

A. Bouville 